1996-02

\title{
Spreading pattern and mesoscale
}

\section{structure of Mediterranean outflow in the}

Iberian Basin estimated from historical

data

Shapiro, Georgy

http://hdl.handle.net/10026.1/9740

10.1016/0924-7963(95)00011-9

Journal of Marine Systems

Elsevier

All content in PEARL is protected by copyright law. Author manuscripts are made available in accordance with publisher policies. Please cite only the published version using the details provided on the item record or document. In the absence of an open licence (e.g. Creative Commons), permissions for further reuse of content should be sought from the publisher or author. 
J O U R N A L F
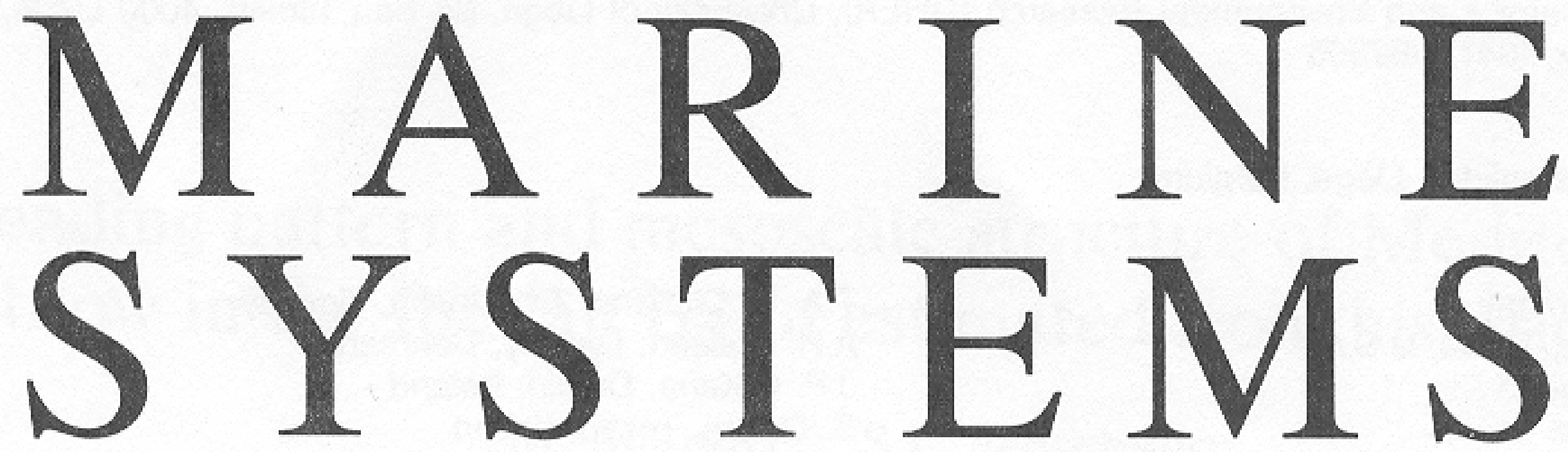

Journal of Marine Systems 7 (1996) 337-348

Spreading pattern and mesoscale structure of Mediterranean outflow in the Iberian Basin estimated from historical data

$$
\text { G.I. Shapiro *, S.L. Meschanov }
$$

P.P. Shirshov Institute of Oceanology, 23 Krasikova Street, 117218 Moscow, Russia

Received 5 August 1994; accepted 14 December 1994

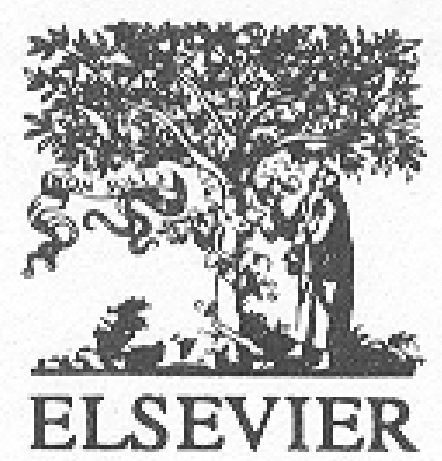




\title{
Spreading pattern and mesoscale structure of Mediterranean outflow in the Iberian Basin estimated from historical data
}

\author{
G.I. Shapiro * , S.L. Meschanov \\ P.P. Shirshov Institute of Oceanology, 23 Krasikova Street, 117218 Moscow, Russia \\ Received 5 August 1994; accepted 14 December 1994
}

\begin{abstract}
The location, spreading pattern, mesoscale structure and the influence of bottom topography on the Mediterranean Water (MW) outflow were studied using historical hydrographic data from the Iberian Basin of the North Atlantic. It was shown on the basis of joint analysis of continuous and discrete component of the MW distribution, that after leaving the Gulf of Cadiz the MW outflow is broken into two main cores: Northwestern and Southwestern ones. The wide, intense Northwestern core spreads in a regular and continuous manner. West off the Tejo plateau it is split into three branches, the most intense of them keeps continuity up to $14^{\circ} \mathrm{W}$. The less intense Southwestern core passes south of the Gettysburg bank and is split into two branches just after the Gulf of Cadiz. This core has a lenticular intermittent character starting with $11^{\circ} \mathrm{W}$. West of $15^{\circ} \mathrm{W}$ all the branches of MW outflow spread in the form of isolated salty patches. In the Iberian Basin $\left(32^{\circ}-44^{\circ} \mathrm{N}, 8^{\circ}-22^{\circ} \mathrm{W}\right) 30 \mathrm{MW}$ lenses were found out, 12 of which were not mentioned in the literature before.
\end{abstract}

\section{Introduction}

The giant salt tongue of modified Mediterranean Water (MW) is an important feature of the hydrography of the Northeastern Atlantic. The MW is formed by mixing the high saline water of the lower current of the Strait of Gibraltar and North Atlantic Central Water (NACW) (Howe, 1982). The estimates of MW discharge vary from 2.6 Sv (Ambar and Howe, 1979) up to 6.5 Sv (Howe, 1984) in different places of the Gulf of Cadiz not only due to time variations but

* Corresponding author. P.P. Shirshov Institute of Oceanology, 23 Krasikova Street, 117218 Moscow, Russia. Tel: +7 (095) 124-7392, Fax: + 7 (095) 124-5983, E-mail: pelevin@glas.apc.org. also to entrainment and mixing with NACW. The MW spreads from the Gulf of Cadiz in the main thermocline at intermediate depths of $500-1500$ $\mathrm{m}$ over many thousands of kilometers. The influence of the high salinity of the MW was detected even in the Indian and Pacific Oceans (Reid, 1978; Richardson et al., 1991).

According to the classical picture, the MW propagates uniformly in radial direction as a continuous water tongue and this process can be described in terms of large scale advective-diffusive balance (Defant, 1955). During the last two decades, field investigations have trustworthy shown that the MW spreads following a much more complex pattern. After leaving the Gulf of Cadiz, the major part of MW flow turns north along the Portugal coast. The result is that the 
climatic salinity maximum at the typical depths of MW spreading is shifted to the north of the latitude of Gibraltar (Richardson et al., 1991). In the eastern boundary layer of the Atlantic near the Portuguese coast the MW tongue is strongly influenced by the bottom relief (Zenk and Armi, 1990; Martins, 1992). According to Zenk and Armi (1990), the MW undercurrent forms two stacked outflow cores with different temperature-salinity characteristics and passes through a broad "gateway" between the Cape St. Vincent and the Gettysburg Bank entering the Iberian Basin. The upper core (depth $750 \mathrm{~m}$ ) shows a strong tendency to follow the contours of the Portuguese continental slope. The lower core (depth $1250 \mathrm{~m}$ ) primarily meanders west and northwestwards. The part of MW undercurrent goes southwestward directed to the Canary Basin (Zenk and Armi, 1990; Käse et al., 1986).

In addition to continuous tongue there are isolated layers, blobs and mesoscale lenses (MEDDIES) of high saline Mediterranean Water (Swallow, 1969; Armi and Zenk, 1984; Krauss and Käse, 1984). Hereafter such structure of the MW outflow will be referred to as a "lenticular" one (Emelianov and Fedorov, 1985). Mediterranean lenses are long-lived features keeping the initial values of temperature and salinity in the core over thousands of kilometers (Belkin et al., 1986; Belkin and Kostianoy, 1988; Richardson et al., 1989). The climatic paths of MEDDY propagation were estimated using spatial distribution of observed lenses (Belkin and Kostianoy, 1988; Richardson et al., 1991), direct measurements of individual lens trajectories over long time (Richardson et al., 1989) and theoretical calculations based on Lagrangean invariant method (Kostianoy and Shapiro, 1989). It was stated that the main lens trajectories are oriented southwestward to the Canary Basin. Nevertheless lenses were observed far to the northwest (Katz, 1970; Richardson et al., 1991) and west off the Strait of Gibraltar (Plakhin and Smirnov, 1982; Käse and Zenk, 1987).

In this paper we study the location, spreading pattern, mesoscale structure and the influence of bottom topography on the Mediterranean Water undercurrent in the Iberian Basin of the North
Atlantic. In contrast to Zenk and Armi (1990) who have restricted themselves to the analysis of data of some selected cruises we use the large data base of historical observations that covers period of several decades and includes individual stations in the deep ocean as well as in the coastal zone of the Iberian Basin. We pay a special attention to the evolution of the main (lower) core of MW while it spreads from the coastal region into the open ocean.

\section{Materials and methods}

The analysis presented in this paper is based on the concept issued by Emelianov and Fedorov (1985) on the lenticular structure of intermediate waters of Mediterranean origin. Developing this concept we use two independent approaches: the study of spatial distribution of (i) continuous tongues of MW and (ii) isolated blobs surrounded by the background mixed Mediterranean and North Atlantic Central Waters. The possibility of the investigation of main flow paths of the MW in the coastal zone of the Iberian Basin using the archived hydrographic data that were collected in different years and conditions is based on the hypothesis by Zenk and Armi (1990). They stated that the bottom topography (underwater canyons, seamounts, continental rise orientation) strongly influences the spatial distribution and structure of MW. Thus, one can expect that along with the stochastic component in the MW distribution caused by synoptic current activity, seasonal and annual variability there is a regular component that geographically correlates to the inhomogenities of the bottom topography.

Our study is based on the materials of individual hydrographic stations taken in the Iberian Basin $\left(32^{\circ}-44^{\circ} \mathrm{N}, 8^{\circ}-22^{\circ} \mathrm{W}\right)$. The data were obtained from National Oceanographic Data Center (NODC, Washington, 1906-1986), Russian National Oceanographic Data Center (WNIIGMI-MCD, Obninsk, 1950-1986) and from several selected cruises listed below.

Only selected stations were retained that were as deep as at least $1250 \mathrm{~m}$ and had the sampling depth spacing less than $500 \mathrm{~m}$. When necessary, 


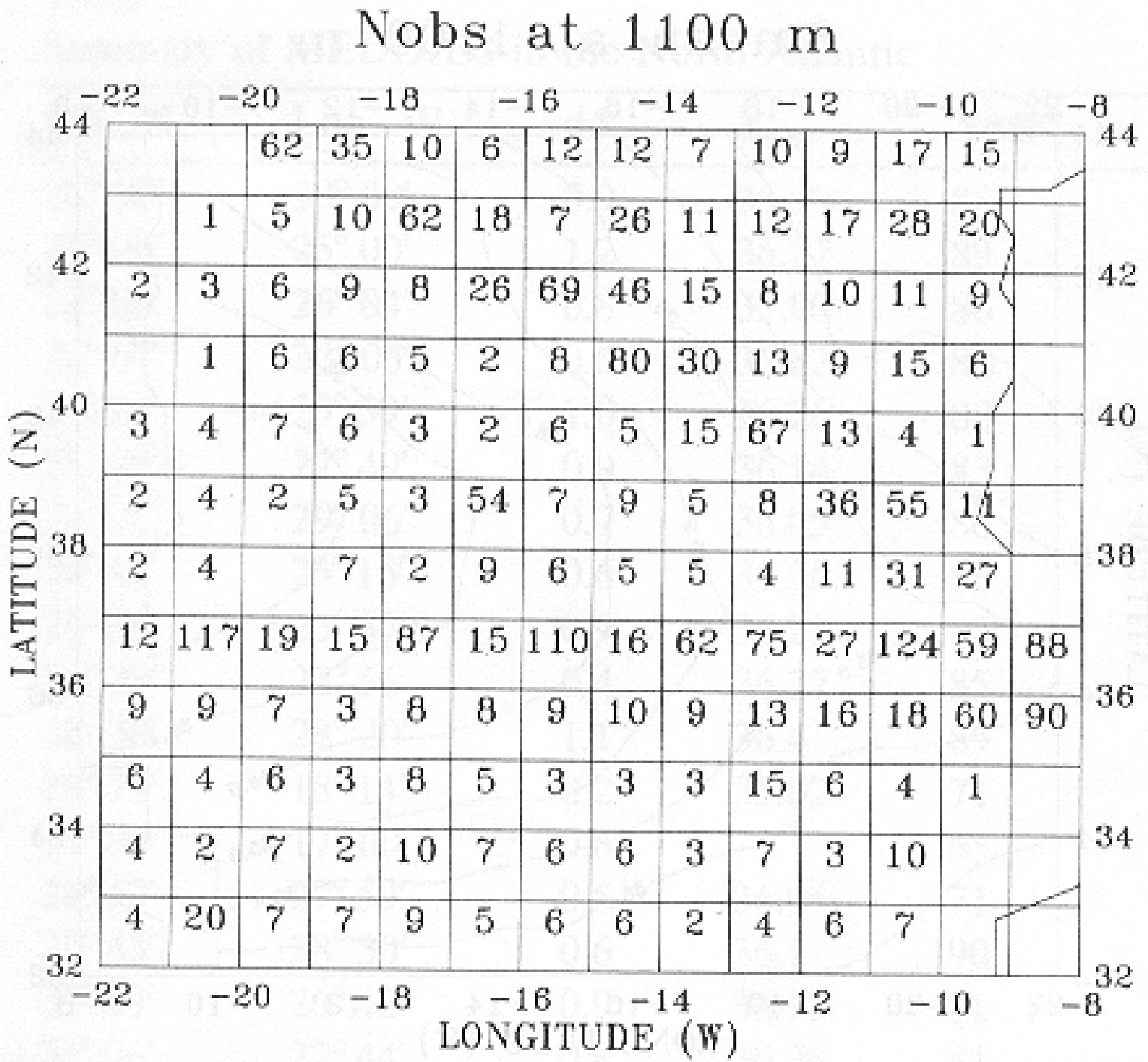

Fig. 1. Distribution of available observations in the Iberian Basin at $1100 \mathrm{~m}$ depth.

the data were interpolated with a $100 \mathrm{~m}$ vertical step in the depth range $500-1300 \mathrm{~m}$ (Table 1). Distribution of hydrographic stations in $1^{\circ} \times 1^{\circ}$ bins at $1100 \mathrm{~m}$ used for the present analysis is shown in Fig. 1. Up to $62 \%$ of them were taken from NODC archive, $38 \%$ from other sources. In common the number of observations Nobs slightly diminishes with depth because some of old stations have gaps in data at deep levels.

To reveal the main paths of MW penetration we use two different statistical methods of data processing: (i) calculation of mean $T, S$ fields in small spatial boxes and (ii) extreme statistics method. We also check visually the individual hydrographic stations with positive salinity anomalies relative to the background values at depths of MW propagation. Below we discuss the salinity field only.

The first method follows mainly the procedure used by Richardson et al. (1991). Mean salinity
$S_{\text {mean }}$ was calculated in each $2^{\circ} \times 2^{\circ}$ box, individual observations with anomaly in salinity exceeding two standard deviations from the mean were eliminated, and the mean recalculated. Successive iterations were continued until consecutive values agreed to within $0.005 \mathrm{psu}$. The resulting grid of salinity values was slightly smoothed using inverse distance method, and maps of $S_{\text {mean }}$ were drawn for levels $500,600,700,800,900,1000$, $1100,1200 \mathrm{~m}$. The mean salinity field reveals a continuous component of MW undercurrent only because it does not take into account rare anomalous stations corresponding to high salinity blobs and lenses.

According to the second method we split the study area into small boxes of different sizes in agreement with the density of observational grid and identify the extreme (in our case - maximal) values of salinity $S_{\max }$ in each box. This approach allows to identify not only areas where the positive anomalies typical for MW are observed repeatedly but also areas where anomalies are met infrequently. Maps of $S_{\max }$ were calculated at the same depth levels as $S_{\text {mean }}$ using adaptive spatial grid. The largest boxes were $1^{\circ} \times 1^{\circ}$ in size. If the number of stations in a box was $30<N_{\mathrm{obs}} \leq 60$ the box was split into smaller $0.5^{\circ} \times 0.5^{\circ}$ boxes, if $N_{\text {obs }}>60-$ into $0.33^{\circ} \times 0.33^{\circ}$ boxes. The usage of adaptive grid with variable step allows to reach the best spatial resolution near the Gulf of Cadiz and the Portuguese coast where the station grid is much denser than in the open ocean. The map of $S_{\max }$ allows to track both continuous and discrete components of MW outflow. If we used an ordinary averaging method the sporadic positive anomalies caused by irregular occurrence of meanders, isolated layers and lenses would be masked by large amount of background data.

Intensity of the discrete component of MW can be estimated using the deviation of maximal

Table 1

Number of observations in the Iberian Basin included in the data base

\begin{tabular}{lrrrrrrrr}
\hline Depth, m & 500 & 600 & 700 & 800 & 900 & 1000 & 1100 & 1200 \\
Nobs (total) & 2643 & 2629 & 2612 & 2616 & 2613 & 2585 & 2573 & 2566 \\
NODC & 1640 & 1636 & 1631 & 1625 & 1623 & 1600 & 1590 & 1586 \\
VNIIGMI-MCD & 1003 & 993 & 981 & 991 & 990 & 985 & 983 & 980 \\
\hline
\end{tabular}


salinity $S_{\max }$ from the background field $S_{\text {mean }}$. In the areas where MW spreads regularly the value of $S_{\text {delta }}=S_{\text {max }}-S_{\text {mean }}$ is close to zero because both maximal and mean values of haline field are formed by the same water (MW). The parameter $S_{\text {delta }}$ reaches its maximum in the areas where the spreading of MW occurs mainly in the form of isolated patches. Therefore, the analysis of spatial distribution of $S_{\text {delta }}$ allows to pick out the regions of preferably continuous and discrete spreading of anomalous water and to locate the regions where the continuous tongue is split into isolated patches.

The independent analysis of discrete component of MW was made using method suggested by Richardson et al. (1991). We selected stations having positive anomalies compared to the local background. The "anomalous" stations were analyzed visually to find vertical profiles typical of MW. For each station with MW presence we found (where possible) the neighbouring stations taken in the same cruise and drew and analyzed vertical sections and/or horizontal salinity charts. The locations of isolated lenses both referred in the literature before and found by ourselves were used to reveal the paths of spreading of discrete component of MW.

\section{Results}

Fig. 2 shows the computed climatic field of $S_{\text {mean }}$ at $1100 \mathrm{~m}$ level. The distribution of $S_{\text {mean }}$ corresponds mainly to the results by Richardson et al. (1991). In the coastal zone the zonal axis of the climatic tongue of $\mathrm{MW}$ with the mean salinity up to $36.2^{\circ}-36.3^{\circ}$ psu is located at $39^{\circ} \mathrm{N}$, i.e. 3 degrees north of the latitude of Gibraltar. The isohaline $36.0 \mathrm{psu}$ that represents the main core of MW tongue goes west as far as $15^{\circ} \mathrm{W}$ both at $1000 \mathrm{~m}$ and $1100 \mathrm{~m}$ levels.

Fig. 3 shows the chart of $S_{\max }$ computed at the same depth level using adaptive spatial grid. The mesoscale distribution of maximal salinity $S_{\max }$ looks strongly different from the mean field. Since the bottom topography is very important in discussion about $S_{\max }$, all the locations mentioned below are mapped in Fig. 8. The high saline MW

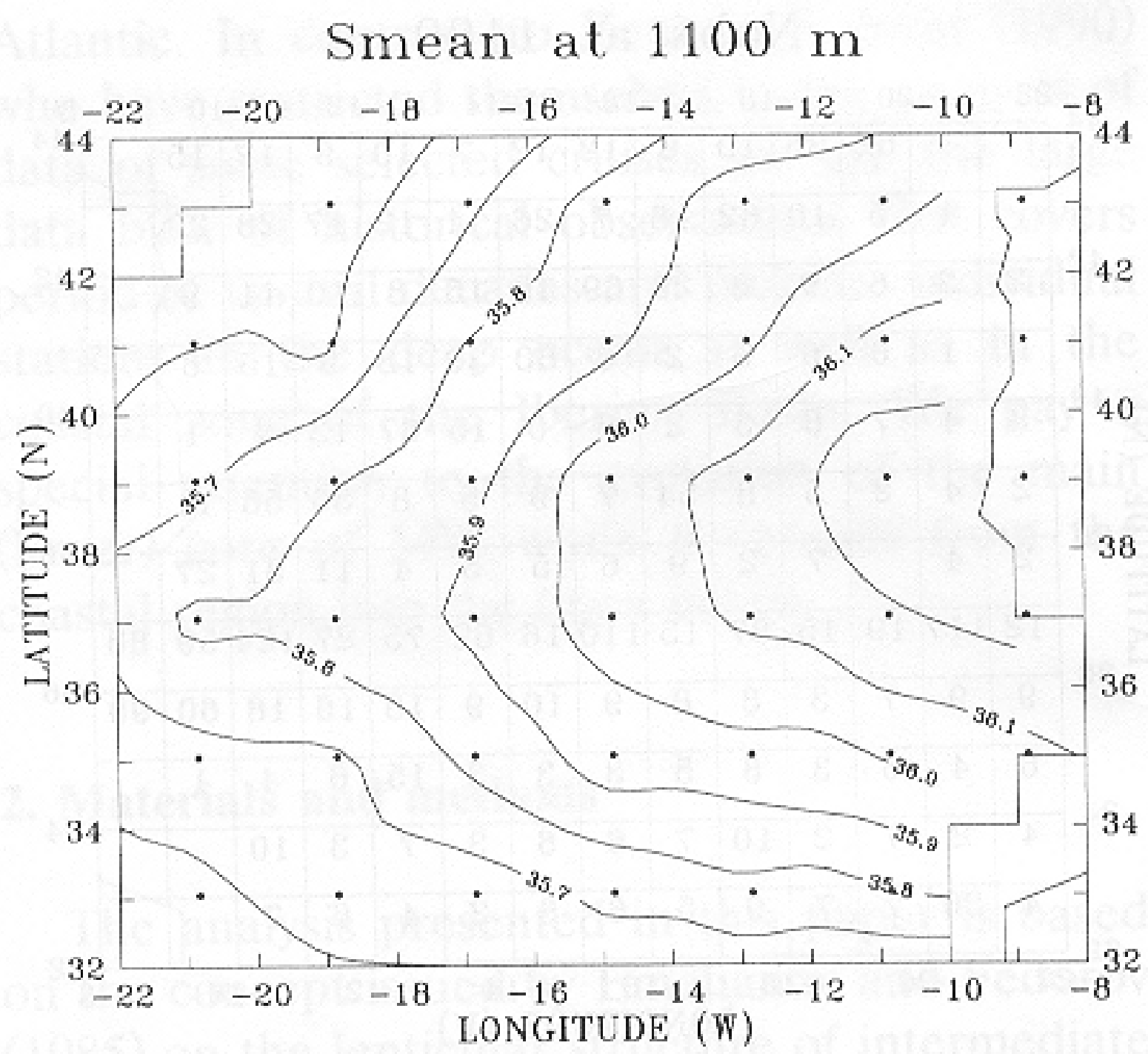

Fig. 2. Contours of the mean salinity field $S_{\text {mean }}$ at $1100 \mathrm{~m}$ depth.

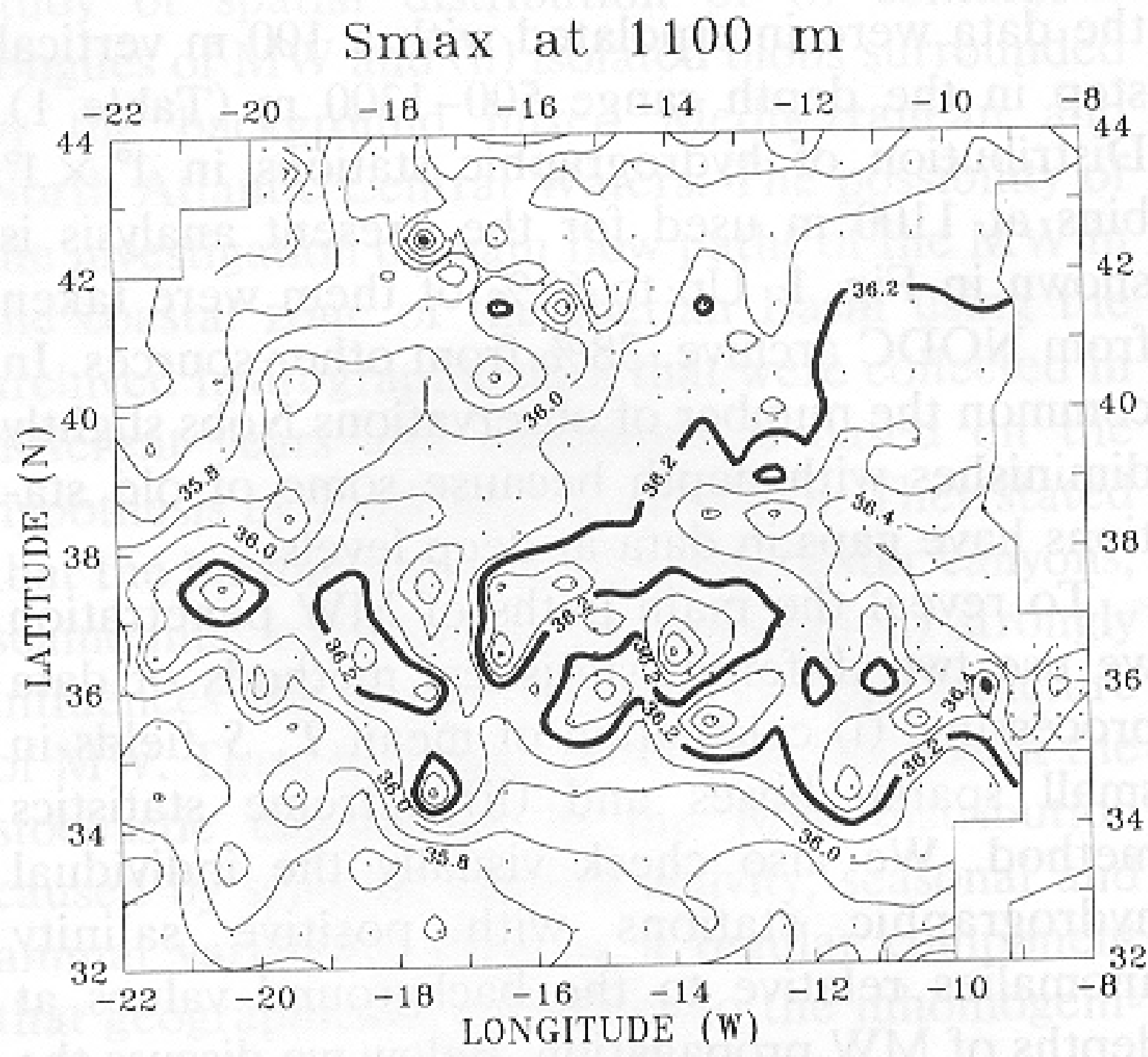

Fig. 3. Contours of the maximal salinity field $S_{\max }$ at $1100 \mathrm{~m}$ depth. Dots mark the nodes of adaptive grid. Isohaline $S_{\max }$ $=36.2$ psu is shown by a thick line.

Table 2

Number of stations with salinity anomalies in the Iberian Basin Richardson's et al. (1991) data are shown in brackets, the bin width is \pm 0.5 psu

\begin{tabular}{lrcrrrll}
\hline $\mathrm{d} S$, psu & 0.2 & 0.3 & 0.4 & 0.5 & 0.6 & 0.7 & 0.8 \\
Total & 380 & $161(16)$ & $84(17)$ & 39 & 10 & 4 & 1 \\
Lenses & 1 & $8(0)$ & $7(17)$ & 6 & 4 & 3 & 1 \\
Undefined & 4 & 7 & 15 & 3 & 1 & 0 & 0 \\
Not lenses & 375 & 146 & 62 & 30 & 5 & 1 & 0 \\
\hline
\end{tabular}


Table 3

Summary of MEDDIES in the North Atlantic

\begin{tabular}{|c|c|c|c|c|c|c|c|c|}
\hline Lat., N & Lon., W & $\mathrm{d} S$ & $S_{\max }$ & Year & Month & Ship & & Reference \\
\hline $20^{\circ} 07^{\prime}$ & $38^{\circ} 09^{\prime}$ & 0.9 & 35.90 & 85 & 06 & Ak.M.Keldysh & & KP \\
\hline $22^{\circ} 00^{\prime}$ & $25^{\circ} 00^{\prime}$ & 1.2 & 36.27 & 89 & $01-03$ & Arnold Veimer & & LL \\
\hline $22^{\circ} 00^{\prime}$ & $26^{\circ} 04^{\prime}$ & 0.8 & 35.90 & 86 & 11 & Meteor & & ZKS; RWASP \\
\hline $22^{\circ} 07^{\prime}$ & $22^{\circ} 06^{\prime}$ & 0.6 & 35.82 & 86 & 10 & Endeavor & $\#$ & - \\
\hline $25^{\circ} 24^{\prime}$ & $25^{\circ} 59^{\prime}$ & 1.0 & 36.26 & 88 & 08 & Oceanus & & MCM \\
\hline $25^{\circ} 40^{\prime}$ & $27^{\circ} 49^{\prime}$ & 0.9 & 36.14 & 83 & 01 & Knorr & \# & W86 \\
\hline $26^{\circ} 32^{\prime}$ & $29^{\circ} 06^{\prime}$ & 0.7 & 36.05 & 80 & $03-04$ & Oceanus & \# & AS \\
\hline $26^{\circ} 45^{\prime}$ & $25^{\circ} 16^{\prime}$ & 0.6 & 35.96 & 88 & 08 & Oceanus & & RMCM \\
\hline $26^{\circ} 55^{\prime}$ & $23^{\circ} 37^{\prime}$ & 0.9 & 36.22 & 85 & 10 & Endeavor & & AHOPRR \\
\hline $28^{\circ} 29^{\prime}$ & $22^{\circ} 56^{\prime}$ & 0.4 & 36.27 & 85 & 06 & Hudson & $\#$ & AHOPRR \\
\hline $28^{\circ} 30^{\prime}$ & $28^{\circ} 20^{\prime}$ & 1.1 & 36.4 & 89 & 03 & Akademik S.Vavilov & & MSF \\
\hline $29^{\circ} 29^{\prime}$ & $18^{\circ} 14^{\prime}$ & 0.2 & 35.62 & 71 & 08 & Elisha Kent Kane & $\#$ & - \\
\hline $29^{\circ} 30^{\prime}$ & $17^{\circ} 00^{\prime}$ & 0.6 & - & 65 & summer & 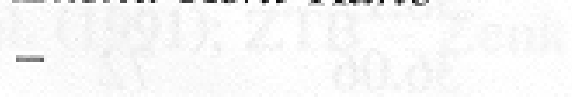 & & $\mathrm{P}$ \\
\hline $29^{\circ} 57^{\prime}$ & $27^{\circ} 59^{\prime}$ & 0.5 & 36.05 & 71 & 01 & Elisha Kent Kane & $\# *$ & RMCM \\
\hline $30^{\circ} 45^{\prime}$ & $28^{\circ} 30^{\prime}$ & 0.6 & 36.1 & 90 & 08 & Akademik Kurchatov & & $\mathrm{ZL}$ \\
\hline $31^{\circ} 00^{\prime}$ & $26^{\circ} 42^{\prime}$ & 0.9 & 36.35 & 81 & 05 & Knorr & & $\mathrm{AZ}$ \\
\hline $31^{\circ} 06^{\prime}$ & $22^{\circ} 44^{\prime}$ & 0.8 & 36.28 & 81 & 06 & Knorr & & $\mathrm{AZ}$ \\
\hline $31^{\circ} 29^{\prime}$ & $11^{\circ} 37^{\prime}$ & 0.9 & 36.29 & 11 & 06 & Mowe & $\#$ & - \\
\hline $31^{\circ} 30^{\prime}$ & $14^{\circ} 27^{\prime}$ & 0.6 & 36.22 & 65 & 10 & - & * & RMCM \\
\hline $31^{\circ} 30^{\prime}$ & $22^{\circ} 35^{\prime}$ & 0.2 & 35.8 & 82 & $03-04$ & Poseidon & & $\mathrm{KZ}$ \\
\hline $31^{\circ} 48^{\prime}$ & $27^{\circ} 00^{\prime}$ & 0.9 & 36.2 & 89 & 11 & Akademik Ioffe & & DMNFS \\
\hline $31^{\circ} 54^{\prime}$ & $27^{\circ} 00^{\prime}$ & 0.9 & 36.35 & 90 & 06 & Vityaz & & - \\
\hline $31^{\circ} 58^{\prime}$ & $21^{\circ} 48^{\prime}$ & 0.8 & 36.30 & 84 & 10 & Oceanus & & AHOPRR \\
\hline $32^{\circ} 13^{\prime}$ & $28^{\circ} 59^{\prime}$ & 0.8 & 36.21 & 90 & 01 & Professor S. Dorofeev & & SME \\
\hline $32^{\circ} 30^{\prime}$ & $11^{\circ} 30^{\prime}$ & 0.6 & - & 65 & summer & - & & $\mathrm{P}$ \\
\hline $32^{\circ} 30^{\prime}$ & $20^{\circ} 30^{\prime}$ & 0.3 & 35.89 & 71 & 04 & Elisha Kent Kane & $\#$ * & $\mathrm{AZ}$ \\
\hline $32^{\circ} 48^{\prime}$ & $12^{\circ} 05^{\prime}$ & 0.8 & 36.58 & 87 & 03 & Professor S. Dorofeev & & VKOP \\
\hline $32^{\circ} 50^{\prime}$ & $10^{\circ} 50^{\prime}$ & - & - & 88 & $06-08$ & Vityaz & & $\mathrm{F}$ \\
\hline $33^{\circ} 00^{\prime}$ & $23^{\circ} 10^{\prime}$ & 0.2 & 35.8 & 82 & $03-04$ & Poseidon & & $\mathrm{KZ}$ \\
\hline $33^{\circ} 08^{\prime}$ & $21^{\circ} 33^{\prime}$ & 0.7 & 36.17 & 81 & 06 & Knorr & & $\mathrm{AZ}$ \\
\hline $33^{\circ} 20^{\prime}$ & $21^{\circ} 25^{\prime}$ & 0.7 & 36.26 & 93 & 10 & Professor Shtokman & & - \\
\hline $33^{\circ} 28^{\prime}$ & $23^{\circ} 20^{\prime}$ & 0.6 & 36.16 & 70 & 11 & Elisha Kent Kane & $\# *$ & $\mathrm{AZ}$ \\
\hline $33^{\circ} 34^{\prime}$ & $10^{\circ} 21^{\prime}$ & 0.4 & 36.00 & 70 & 11 & Elisha Kent Kane & $\# *$ & RMCM \\
\hline $33^{\circ} 55^{\prime}$ & $24^{\circ} 05^{\prime}$ & 1.1 & 36.56 & 85 & 11 & Endeavor & & RWASP \\
\hline $33^{\circ} 56^{\prime}$ & $20^{\circ} 19^{\prime}$ & 0.4 & 35.99 & 80 & 11 & Musson & \# & - \\
\hline $34^{\circ} 00^{\prime}$ & $13^{\circ} 00^{\prime}$ & 0.5 & 36.33 & 66 & 06 & - & ${ }^{*}$ & RMCM \\
\hline $34^{\circ} 00^{\prime}$ & $22^{\circ} 38^{\prime}$ & 0.7 & 36.22 & 89 & $01-03$ & Arnold Veimer & & $\mathrm{LL}$ \\
\hline $34^{\circ} 01^{\prime}$ & $17^{\circ} 01^{\prime}$ & 0.7 & 36.44 & 65 & 09 & Origny & \#* & RMCM \\
\hline $34^{\circ} 15^{\prime}$ & $11^{\circ} 30^{\prime}$ & - & - & 88 & $06-08$ & Vityaz & & $\mathrm{F}$ \\
\hline $34^{\circ} 34^{\prime}$ & $11^{\circ} 04^{\prime}$ & 0.6 & 36.57 & 71 & 09 & Elisha Kent Kane & * & RMCM \\
\hline $34^{\circ} 35^{\prime}$ & $9^{\circ} 00^{\prime}$ & - & - & 88 & $06-08$ & Vityaz & & $\mathrm{F}$ \\
\hline $34^{\circ} 38^{\prime}$ & $19^{\circ} 42^{\prime}$ & 0.4 & 36.11 & 71 & 07 & Sands & $\# *$ & RMCM \\
\hline $34^{\circ} 39^{\prime}$ & $24^{\circ} 19^{\prime}$ & 0.6 & 36.20 & 83 & 06 & Discovery & $\#$ & - \\
\hline $34^{\circ} 44^{\prime}$ & $23^{\circ} 29^{\prime}$ & - & 36.53 & 92 & 03 & Charles Darwin & & PLC \\
\hline $34^{\circ} 49^{\prime}$ & $21^{\circ} 12^{\prime}$ & 0.7 & 35.95 & 90 & 03 & Akademik Ioffe & & BMRS \\
\hline $35^{\circ} 00^{\prime}$ & $10^{\circ} 00^{\prime}$ & 0.6 & 36.65 & 70 & 08 & Rockaway & $\# *$ & RMCM \\
\hline $35^{\circ} 00^{\prime}$ & $26^{\circ} 30^{\prime}$ & 0.5 & 35.90 & 67 & 07 & Gillis & \#* & $\mathrm{AZ}$ \\
\hline $35^{\circ} 09^{\prime}$ & $13^{\circ} 22^{\prime}$ & 0.5 & 36.42 & 48 & 01 & Atlantis-I & \#* & $\mathrm{RMCM}$ \\
\hline $35^{\circ} 26^{\prime}$ & $10^{\circ} 00^{\prime}$ & - & - & 88 & 09 & Oceanus & & KPS \\
\hline $35^{\circ} 30^{\prime}$ & $9^{\circ} 00^{\prime}$ & 0.8 & 36.74 & 82 & 09 & Ernst Krenkel & \# & - \\
\hline $35^{\circ} 30^{\prime}$ & $13^{\circ} 00^{\prime}$ & - & - & 88 & $06-08$ & Vityaz & & $\mathrm{F}$ \\
\hline $35^{\circ} 30^{\prime}$ & $17^{\circ} 00^{\prime}$ & 0.4 & 36.20 & 88 & 03 & Poseidon & & KBH; SHK \\
\hline
\end{tabular}


Table 3 (continued)

\begin{tabular}{|c|c|c|c|c|c|c|c|c|}
\hline Lat., N & Lon., W & $\mathrm{d} S$ & $S_{\max }$ & Year & Month & Ship & & Reference \\
\hline $35^{\circ} 45^{\prime}$ & $8^{\circ} 57^{\prime}$ & 0.3 & 36.55 & 58 & 11 & Discovery-II & \# & $S$ \\
\hline $35^{\circ} 52^{\prime}$ & $10^{\circ} 36^{\prime}$ & - & - & 88 & 09 & Oceanus & & KPS \\
\hline $35^{\circ} 52^{\prime}$ & $13^{\circ} 33^{\prime}$ & 0.6 & 36.55 & 51 & 02 & San Pablo & \# & - \\
\hline $35^{\circ} 58^{\prime}$ & $13^{\circ} 59^{\prime}$ & 0.6 & 36.50 & 83 & 03 & Ernst Krenkel & \# & - \\
\hline $36^{\circ} 00^{\prime}$ & $13^{\circ} 30^{\prime}$ & 0.8 & 36.5 & 85 & 09 & Akademik Kurchatov & & $\mathrm{ZO}$ \\
\hline $36^{\circ} 00^{\prime}$ & $17^{\circ} 30^{\prime}$ & 0.5 & 36.45 & 83 & 01 & Viktor Bugaev & $\#$ & - \\
\hline $36^{\circ} 00^{\prime}$ & $20^{\circ} 00^{\prime}$ & 0.4 & 36.19 & 84 & 03 & Ernst Krenkel & \# & - \\
\hline $36^{\circ} 00^{\prime}$ & $20^{\circ} 40^{\prime}$ & 0.4 & 36.1 & 82 & $03-04$ & Poseidon & & $\mathrm{KZ}$ \\
\hline $36^{\circ} 00^{\prime}$ & $22^{\circ} 30^{\prime}$ & 0.5 & 36.21 & 79 & 09 & Musson & \# & - \\
\hline $36^{\circ} 00^{\prime}$ & $22^{\circ} 30^{\prime}$ & 0.8 & 36.51 & 83 & 08 & Ernst Krenkel & \# & - \\
\hline $36^{\circ} 00^{\prime}$ & $24^{\circ} 00^{\prime}$ & 0.7 & 36.30 & 83 & 04 & Viktor Bugaev & \# & - \\
\hline $36^{\circ} 00^{\prime}$ & $24^{\circ} 30^{\prime}$ & 0.4 & 35.91 & 67 & 03 & - & $"$ & RMCM \\
\hline $36^{\circ} 00^{\prime}$ & $27^{\circ} 31^{\prime}$ & 0.6 & 36.17 & 77 & 07 & Musson & \# & - \\
\hline $36^{\circ} 00^{\prime}$ & $29^{\circ} 58^{\prime}$ & 0.6 & 36.06 & 72 & 04 & Poryv & $\#$ & - \\
\hline $36^{\circ} 00^{\prime}$ & $30^{\circ} 00^{\prime}$ & 0.7 & 36.18 & 76 & 04 & Ernst Krenkel & $\#$ & - \\
\hline $36^{\circ} 00^{\prime}$ & $30^{\circ} 00^{\prime}$ & 0.6 & 36.42 & 76 & 06 & Ernst Krenkel & & PS82 \\
\hline $36^{\circ} 01^{\prime}$ & $17^{\circ} 30^{\prime}$ & 0.7 & 36.63 & 81 & 04 & Ernst Krenkel & \# & - \\
\hline $36^{\circ} 06^{\prime}$ & $9^{\circ} 12^{\prime}$ & - & - & 88 & 09 & Oceanus & & KPS; PS92 \\
\hline $36^{\circ} 13^{\prime}$ & $8^{\circ} 03^{\prime}$ & 0.8 & 36.6 & 71 & 03 & Discovery & & $\mathrm{T}$ \\
\hline $36^{\circ} 14^{\prime}$ & $11^{\circ} 53^{\prime}$ & 0.5 & 36.48 & 81 & 07 & Atlantis-II & \# & RW \\
\hline $36^{\circ} 23^{\prime}$ & $10^{\circ} 00^{\prime}$ & 0.4 & 36.34 & 71 & 01 & Elisha Kent Kane & \#* & $\mathrm{RMCM}$ \\
\hline $36^{\circ} 25^{\prime}$ & $8^{\circ} 35^{\prime}$ & 0.5 & 36.5 & 73 & 03 & Shackleton & & HAD \\
\hline $36^{\circ} 30^{\prime}$ & $14^{\circ} 45^{\prime}$ & 0.4 & 36.30 & 71 & 05 & Elisha Kent Kane & $\# *$ & RMCM \\
\hline $36^{\circ} 35^{\prime}$ & $12^{\circ} 30^{\prime}$ & 0.7 & 36.48 & 92 & 03 & Charles Darwin & & PLC \\
\hline $36^{\circ} 48^{\prime}$ & $10^{\circ} 20^{\prime}$ & 0.4 & 36.51 & 56 & 06 & President T.Tissier & \#* & RMCM \\
\hline $36^{\circ} 50^{\prime}$ & $12^{\circ} 00^{\prime}$ & 0.6 & 36.55 & 88 & 03 & Poseidon & & KBH; SHK \\
\hline $37^{\circ} 00^{\prime}$ & $19^{\circ} 00^{\prime}$ & 0.8 & - & 85 & $02-03$ & Arnold Veimer & & E \\
\hline $37^{\circ} 00^{\prime}$ & $20^{\circ} 01^{\prime}$ & 0.4 & 36.16 & 88 & 08 & Oceanus & & RMCM \\
\hline $37^{\circ} 01^{\prime}$ & $10^{\circ} 14^{\prime}$ & 0.5 & 36.45 & 58 & 04 & - & $*$ & RMCM \\
\hline $37^{\circ} 04^{\prime}$ & $16^{\circ} 42^{\prime}$ & 0.5 & 36.41 & 83 & 06 & Discovery & $\#$ & - \\
\hline $37^{\circ} 04^{\prime}$ & $18^{\circ} 48^{\prime}$ & 0.4 & 36.15 & 31 & 06 & Dana & \#* & RMCM \\
\hline $37^{\circ} 08^{\prime}$ & $10^{\circ} 19^{\prime}$ & 0.5 & 36.51 & 57 & 10 & Crawford & $\# *$ & RMCM \\
\hline $37^{\circ} 28^{\prime}$ & $20^{\circ} 28^{\prime}$ & 0.9 & 36.56 & 71 & 04 & Elisha Kent Kane & $\# *$ & RMCM \\
\hline $37^{\circ} 29^{\prime}$ & $21^{\circ} 28^{\prime}$ & 0.4 & 36.08 & 71 & 07 & Sands & $\# *$ & RMCM \\
\hline $37^{\circ} 54^{\prime}$ & $9^{\circ} 54^{\prime}$ & - & - & 91 & 11 & Sonne & & ZTB \\
\hline $38^{\circ} 00^{\prime}$ & $12^{\circ} 05^{\prime}$ & 0.4 & 36.52 & 70 & 05 & Gemma & \# & - \\
\hline $38^{\circ} 00^{\prime}$ & $12^{\circ} 48^{\prime}$ & - & - & 91 & $04-05$ & - & & ST \\
\hline $38^{\circ} 22^{\prime}$ & $13^{\circ} 07^{\prime}$ & 0.4 & 36.49 & 30 & 08 & Albacora & \# * & RMCM \\
\hline $38^{\circ} 30^{\prime}$ & $13^{\circ} 20^{\prime}$ & - & - & 91 & $04-05$ & - & & ST \\
\hline $38^{\circ} 51^{\prime}$ & $18^{\circ} 17^{\prime}$ & 0.3 & 36.15 & 71 & 06 & Sands & $\#$ & - \\
\hline $39^{\circ} 30^{\prime}$ & $15^{\circ} 00^{\prime}$ & 0.6 & 36.27 & 88 & 03 & Poseidon & & KBH; SHK \\
\hline $39^{\circ} 50^{\prime}$ & $14^{\circ} 30^{\prime}$ & 0.4 & 36.27 & 89 & 06 & Poseidon & & HRKZ \\
\hline $40^{\circ} 05^{\prime}$ & $12^{\circ} 48^{\prime}$ & 0.6 & 36.45 & 89 & 06 & Poseidon & & HRKZ \\
\hline $40^{\circ} 30^{\prime}$ & $12^{\circ} 00^{\prime}$ & 0.7 & 36.30 & 88 & 03 & Poseidon & & KBH; SHK \\
\hline $40^{\circ} 40^{\prime}$ & $26^{\circ} 16^{\prime}$ & 0.6 & 36.02 & 49 & 10 & - & * & $\mathrm{RMCM}$ \\
\hline $41^{\circ} 13^{\prime}$ & $11^{\circ} 30^{\prime}$ & 0.4 & 36.28 & 89 & 01 & Meteor & & ZMW \\
\hline $41^{\circ} 14^{\prime}$ & $13^{\circ} 08^{\prime}$ & 0.3 & 36.24 & 70 & 08 & Rockaway & $\#$ & - \\
\hline $41^{\circ} 30^{\prime}$ & $27^{\circ} 40^{\prime}$ & 0.2 & 35.37 & 68 & $08-09$ & Chain & & $\mathrm{K}$ \\
\hline $42^{\circ} 00^{\prime}$ & $29^{\circ} 15^{\prime}$ & 0.2 & 35.42 & 68 & $08-09$ & Chain & & K \\
\hline $42^{\circ} 53^{\prime}$ & $29^{\circ} 20^{\prime}$ & 0.2 & 35.46 & 68 & $08-09$ & Chain & & $\mathrm{K}$ \\
\hline $42^{\circ} 34^{\prime}$ & $17^{\circ} 30^{\prime}$ & 0.5 & 36.23 & 81 & 01 & Passat & $\#$ & - \\
\hline $43^{\circ} 10^{\prime}$ & $29^{\circ} 15^{\prime}$ & 0.2 & 35.46 & 68 & $08-09$ & Chain & & $\mathrm{K}$ \\
\hline $43^{\circ} 32^{\prime}$ & $24^{\circ} 45^{\prime}$ & 0.4 & 35.57 & 22 & 06 & - & $*$ & RMCM \\
\hline $44^{\circ} 00^{\prime}$ & $17^{\circ} 59^{\prime}$ & 0.6 & 36.06 & 71 & 09 & Elisha Kent Kane & $\# *$ & RMCM \\
\hline
\end{tabular}


Table 3 (continued)

\begin{tabular}{llllllll}
\hline Lat., N & Lon., W & $\mathrm{d} S$ & $S_{\max }$ & Year & Month & Ship & Reference \\
\hline $44^{\circ} 02^{\prime}$ & $17^{\circ} 59^{\prime}$ & 0.6 & 36.06 & 68 & 10 & - & RMCM \\
$45^{\circ} 07^{\prime}$ & $15^{\circ} 56^{\prime}$ & 0.4 & 35.99 & 58 & 09 & - & $*$ \\
$46^{\circ} 00^{\prime}$ & $22^{\circ} 00^{\prime}$ & 0.2 & 35.5 & 58 & 09 & Discovery-II & RMCM \\
\hline
\end{tabular}

* Found by Armi and Zenk (1984) and/or Richardson et al. (1991) using historical data.

\# Found in the present paper using historical data.

Abbreviations in Table 3: AS - Armi and Stommel (1983); AZ - Armi and Zenk (1984); AHOPRR - Armi et al. (1989); BMRS Berezutsky et al. (1993); DMNFS - Dykhno et al. (1991); E - Elken (1987); F - Filyushkin (1989); HAD - Howe et al. (1974); H Harvey (1982); HRKZ - Hinrichsen et al. (1993); K - Katz (1970); KZ - Käse and Zenk (1987); KP - Koshliakov and Panteleev (1988); KBH - Käse et al. (1989); KPS - Kenelly et al. (1989); LL - Laanemets and Lips (1991); MSF - Maltsev et al. (1990); P Piip (1969); PLC - Pingree and Le Cann (1993); PS82 - Plakhin and Smirnov (1982); PS92 - Prater and Sanford (1992); RW Roemmich and Wunsch (1985); RWASP - Richardson et al. (1989); RMCM - Richardson et al. (1991); S - Swallow (1969); SHK Stammer et al. (1991); SME - Shapiro et al. (1992); ST - Shultz Tokos (1992); T - Thorpe (1976); VKOP - Vorobyov et al. (1988); W86 - Williams et al. (1986); ZMW - Zenk et al. (1989); ZKS - Zenk et al. (1991); ZTB - Zenk et al. (1992); ZL - Zhurbas and Lozovatsky (1991); ZO - Zubin and Ozmidov (1987).

outflow $\left(S_{\max }>36.2 \mathrm{psu}\right)$ is broken up into two basic cores. The main core passes between the Cape St. Vincent and the Gettysburg bank northwestward in agreement with the results by Zenk and Armi (1990). One part of the core goes round the Tejo plateau, another one turns west, skirts the northern part of the Ashton seamount and splits into two branches. The first(northwest) branch crosses the Iberian abyssal plane and forms several patches of saline water at $41^{\circ}-44^{\circ}$ $\mathrm{N}$ after passing over small unnamed peaks shown

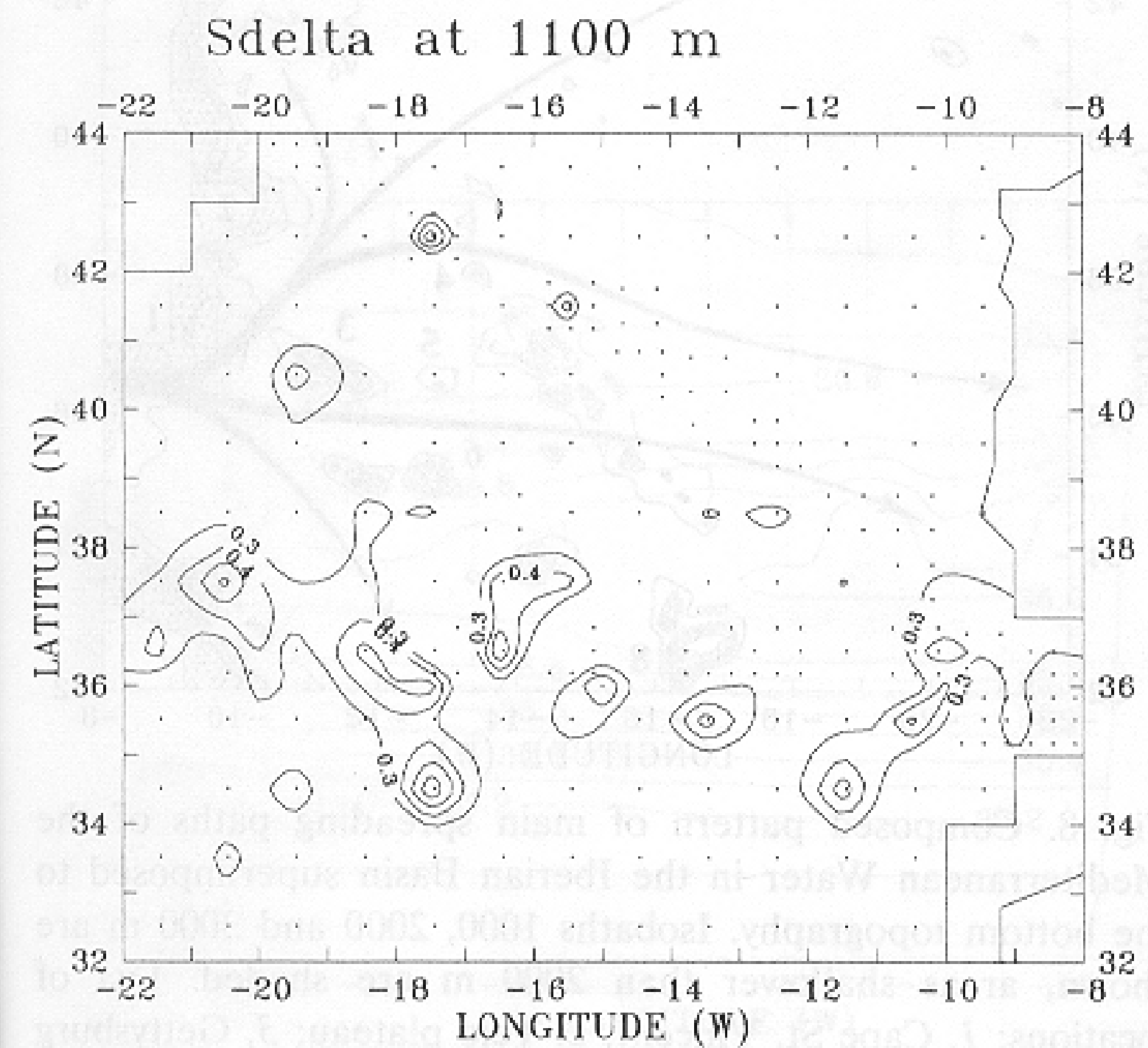

Fig. 4. Contours of the salinity anomaly field $S_{\text {delta }} \geq 0.3$ psu at $1100 \mathrm{~m}$ depth. in Fig. 8. The second (western) branch skirts the Josephine seamount and turns west-southwestward. It is split into isolated patches of more saline water in the latitude band of $36^{\circ}-38^{\circ} \mathrm{N}$.

The second of the two basic cores spreads from the Cape St. Vincent southwestward through the passage between the Gettysburg bank and the Ampere seamount. It skirts the southern peripheries of the Hirondelle and Josephine seamounts. This core is not so wide as the main one. It

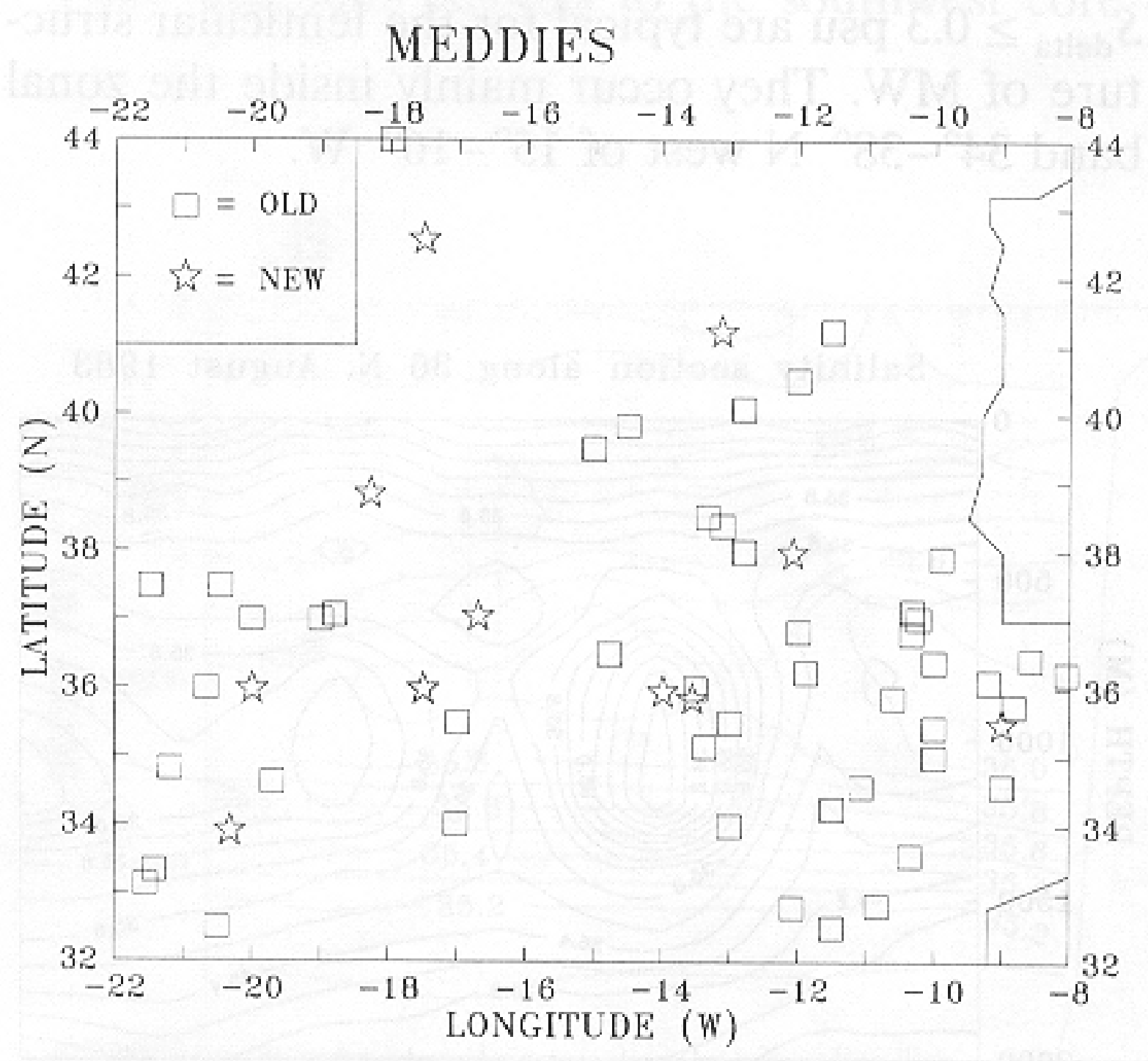

Fig. 5. Locations of MEDDIES in the Iberian Basin based on Table 3. OLD - MEDDIES reported in the literature before; NEW - MEDDIES found in this paper using the archival data set and not reported in the literature before. 


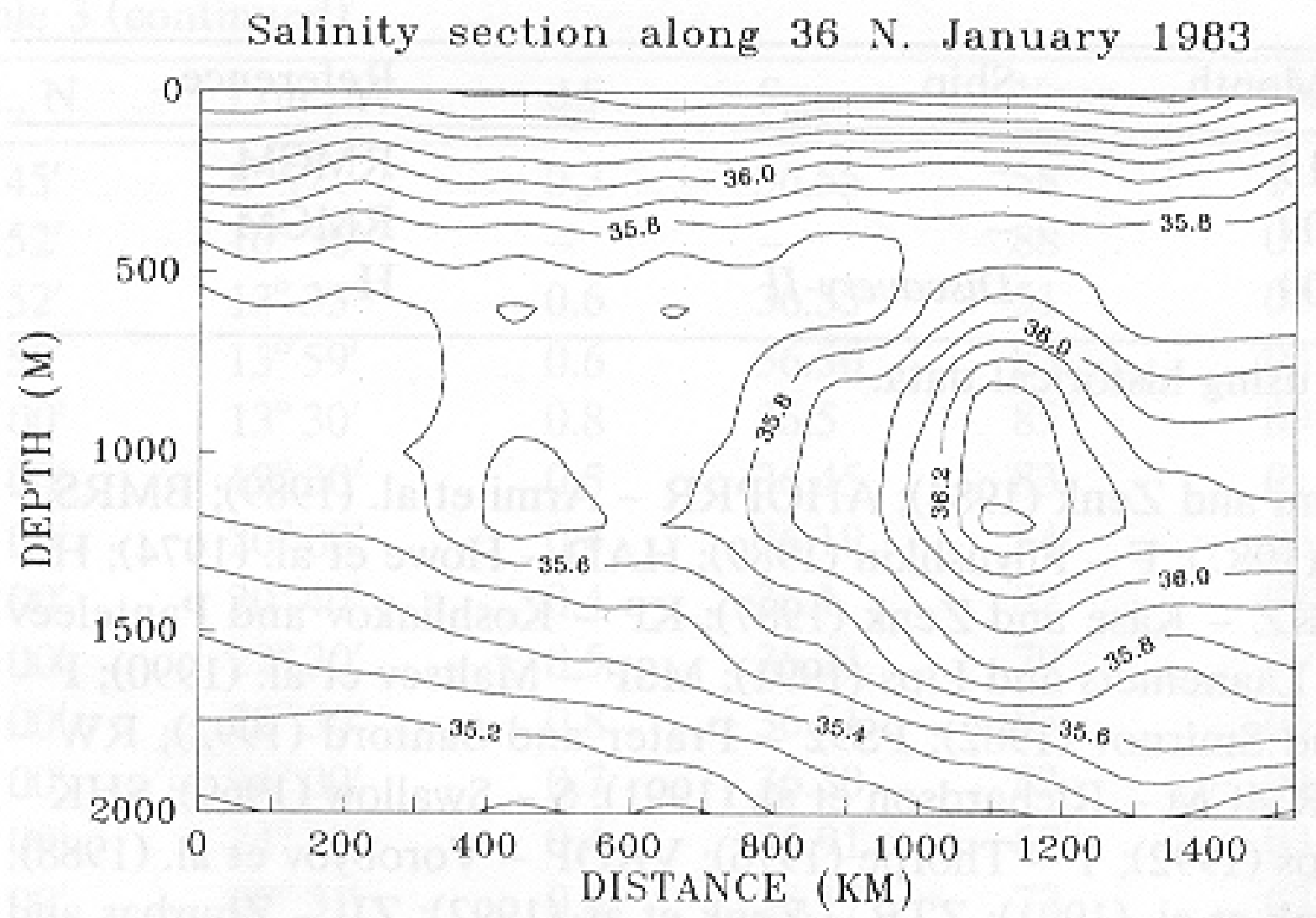

Fig. 6. Salinity cross section through the lens observed by $\mathrm{R} / \mathrm{V}$ Viktor Bugaev. The lens is centered at $36^{\circ} \mathrm{N}, 17^{\circ} 30^{\prime} \mathrm{W}$.

becomes intermittent earlier (approx. since $12^{\circ}$ $\mathrm{W})$. West of $16^{\circ} \mathrm{W}$ it becomes fully discrete and probably joins the main northwest core. Fig. 3 shows also a tendency for the southwest core to be split forming a more southern branch directed from the Cape St. Vincent to the Canary Basin.

Fig. 4. shows the distribution of $S_{\text {delta }}$ at 1100 m. Small values of $S_{\text {delta }}$ correspond to continuous, regular spreading of MW. The main northwest core is continuous east of $16^{\circ} \mathrm{W}$ where typical values of $S_{\text {delta }}<0.3 \mathrm{psu}$. The values of $S_{\text {delta }} \geq 0.3$ psu are typical for the lenticular structure of MW. They occur mainly inside the zonal band $34^{\circ}-38^{\circ} \mathrm{N}$ west of $15^{\circ}-16^{\circ} \mathrm{W}$.

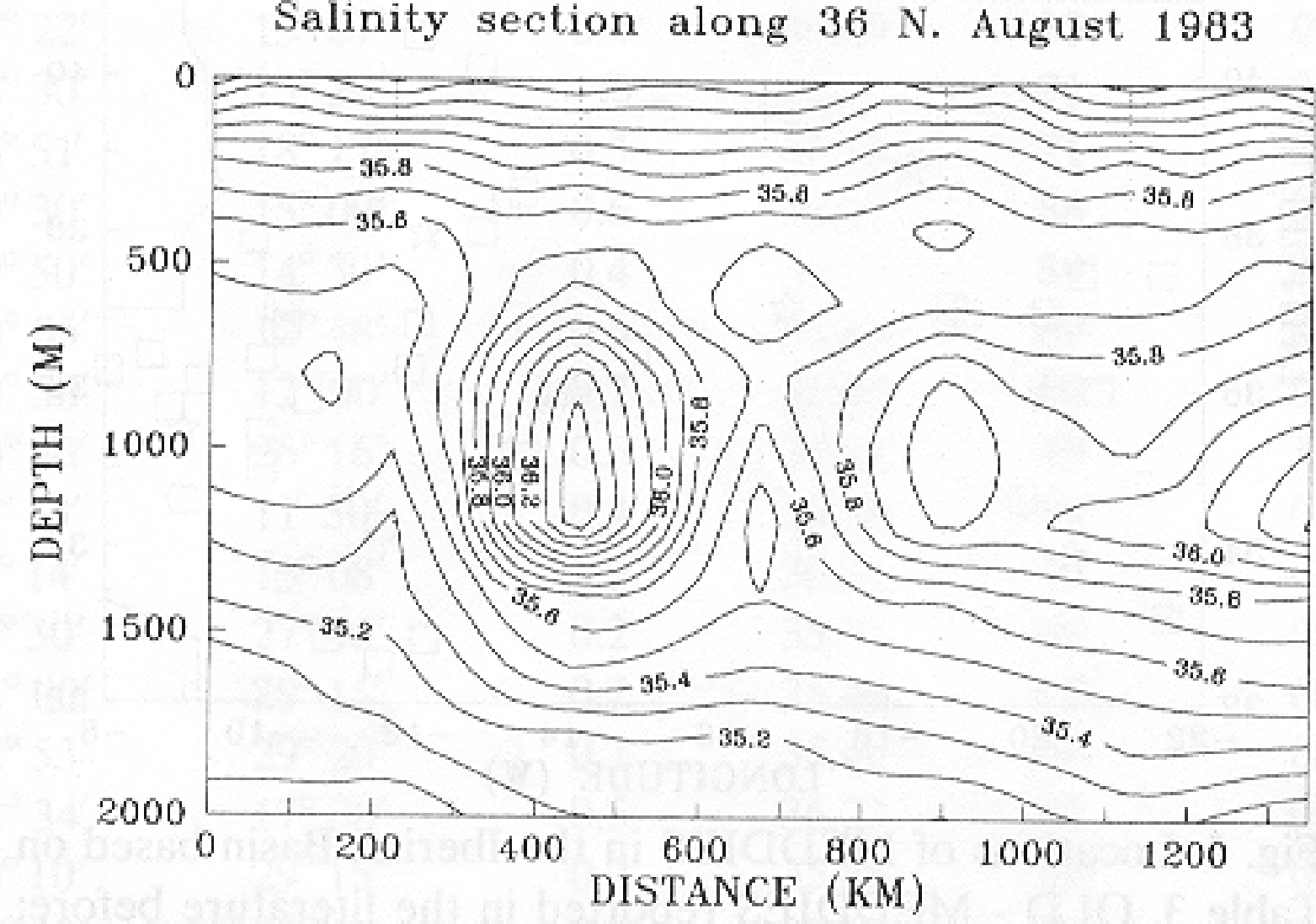

Fig. 7. Salinity cross section through the lens observed by $\mathrm{R} / \mathrm{V}$ Ernst Krenkel. The lens is centered at $36^{\circ} \mathrm{N}, 22^{\circ} 30^{\prime} \mathrm{W}$.
The southward band of high $S_{\text {delta }}$ values near the Cape St. Vincent shows that the south branch of the southwest core of MW outflow consists mainly of isolated patches and lenses.

The discrete component of MW outflow was studied in more detail by analyzing individual anomalous stations and the areas around. The number of stations having positive salinity anomalies $\mathrm{d} S=\left(S-S_{\text {mean }}\right)$ of $0.2-0.8$ psu and thickness of an anomalous layer not less than $200 \mathrm{~m}$ is presented in Table 2. Based on vertical salinity sections we identify thirty lenses of Mediterranean Water (MEDDIES) in our data base. To our knowledge, twelve of them were not mentioned in the literature before. It was found that 619 anomalous stations were not taken in MEDDIES. We had not enough data to distinguish trustworthy the other thirty stations.

We carried out a similar search for the larger area of Eastern North Atlantic $\left(20^{\circ}-50^{\circ} \mathrm{N}, 8^{\circ}-\right.$ $\left.30^{\circ} \mathrm{W}\right)$. We found the total amount of $46 \mathrm{MED}-$ DIES, 22 of which were not reported in the literature. The results of our search together with the lenses reported earlier are listed in Table 3.

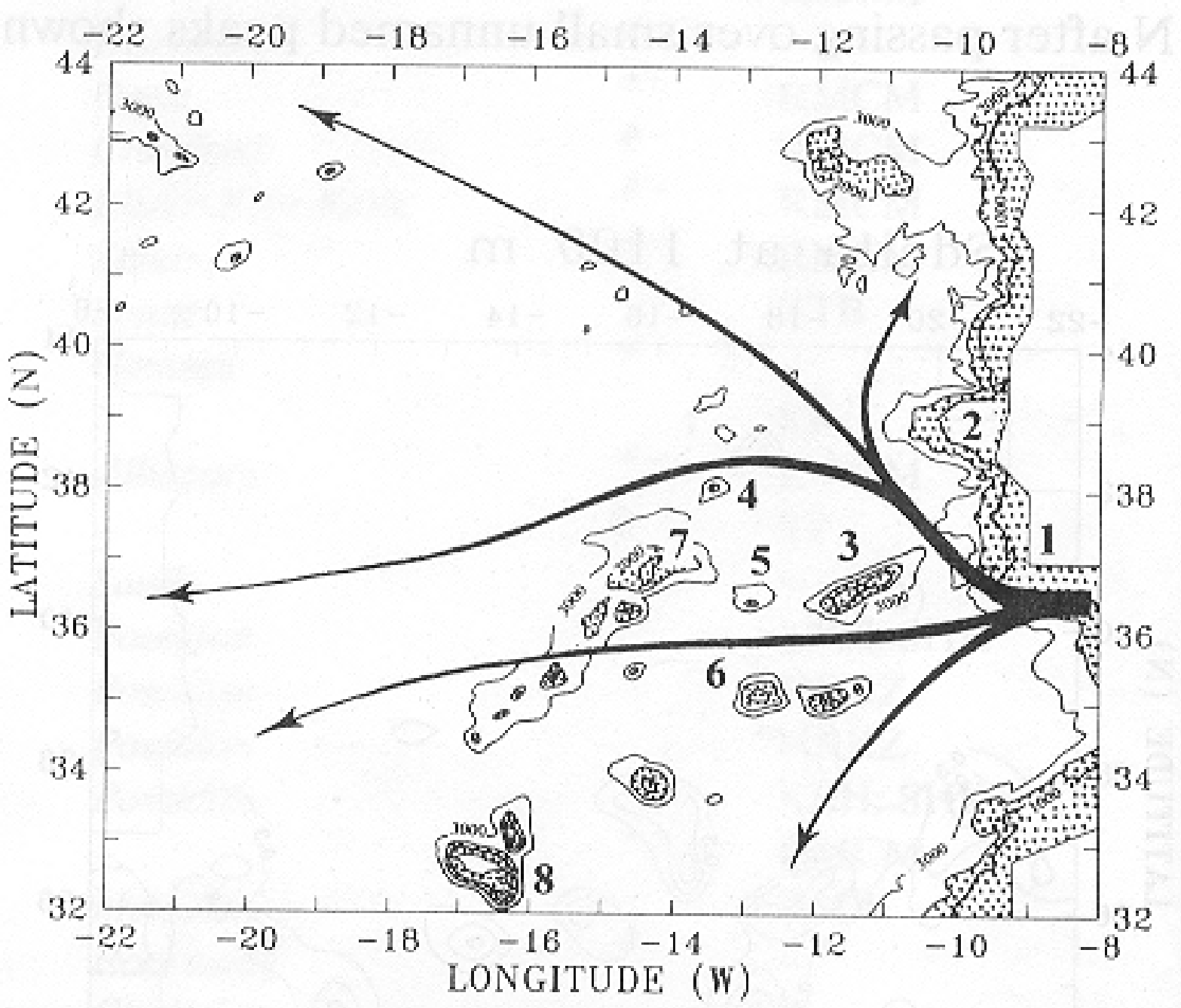

Fig. 8. Composed pattern of main spreading paths of the Mediterranean Water in the Iberian Basin superimposed to the bottom topography. Isobaths 1000,2000 and $3000 \mathrm{~m}$ are shown, areas shallower than $2000 \mathrm{~m}$ are shaded. List of locations: 1. Cape St. Vincent; 2. Tejo plateau; 3. Gettysburg bank; 4. Ashton seamount; 5 . Hirondelle seamount; 6 . Ampere seamount; 7. Josephine seamount; 8 . Madeira Islands. 

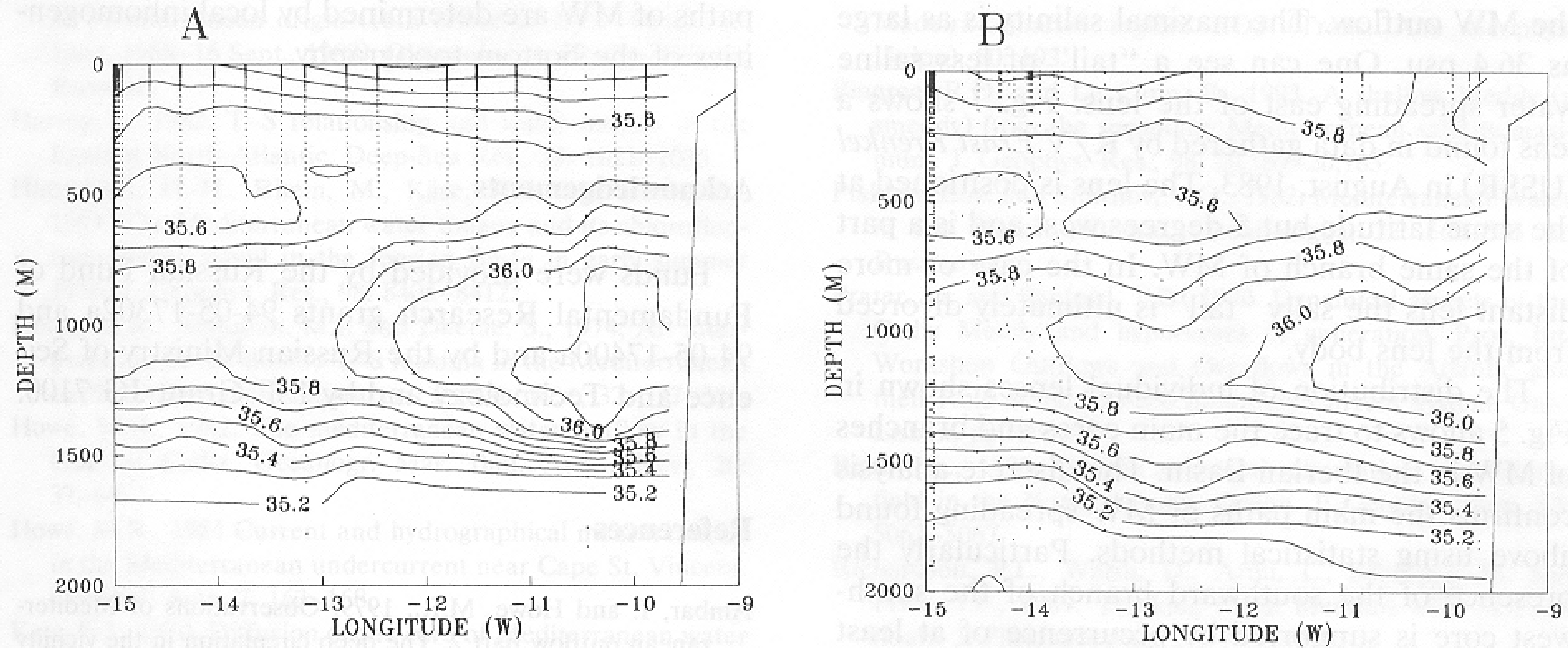

Fig. 9. Salinity cross sections along $41^{\circ} \mathrm{N}$ off the Portuguese continental slope: (a) R/V Elisha Kent Kane (Nov. 1972); (b) archival data (1914-1982).

This Table is more complete than published before (Belkin et al., 1986; Belkin and Kostianoy, 1988; Richardson et al., 1991). The locations of the lenses in the Iberian Basin are shown in Fig. 5 .

The number of lenses is less than the number of anomalous stations because of two reasons. First, normally there are several stations in the same lens. Second, many stations with small values of $\mathrm{d} S$ were taken not in lenses but in the meanders of continuous large scale MW tongue.

Examples of individual lenses found in historical data are shown in Figs. 6, 7. Fig. 6 shows the lens revealed in hydrographic survey data gathered by R/V Viktor Bugaev (USSR) in January 1983. The lens belongs to the southwest core of
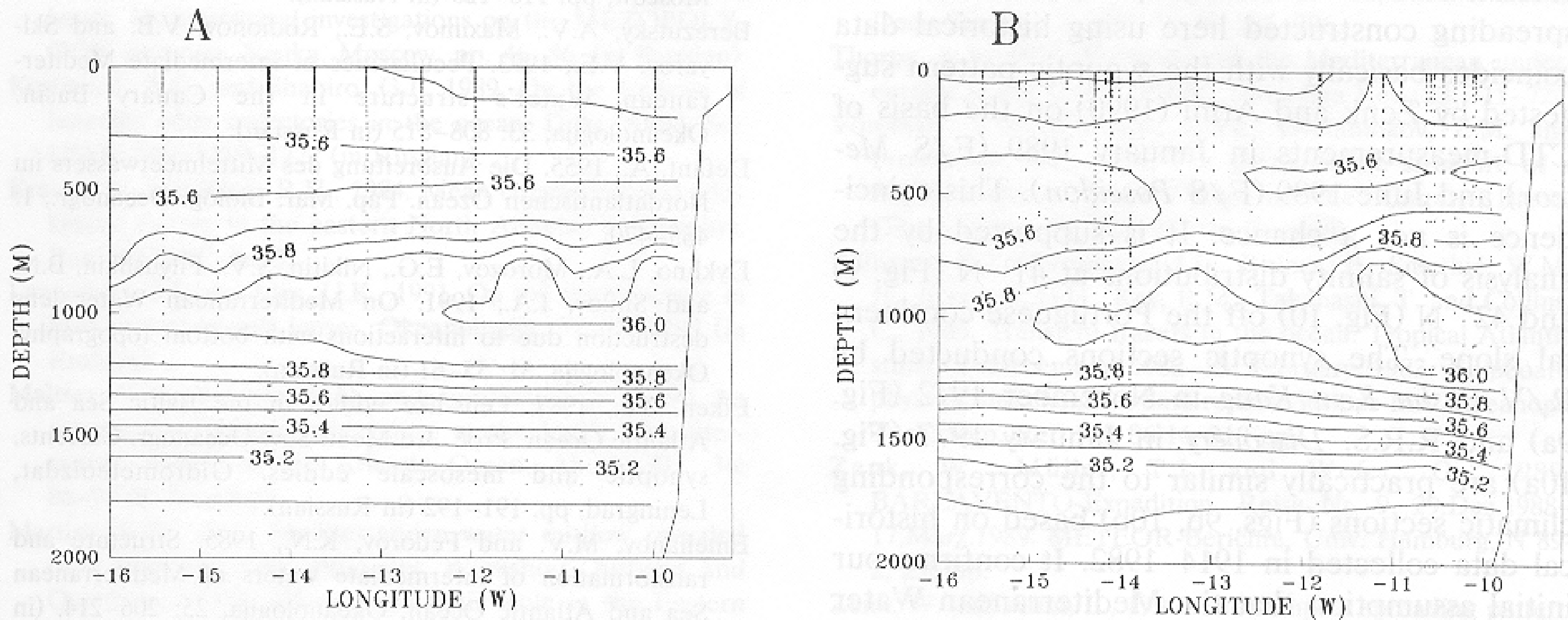

Fig. 10. Salinity cross sections along $42^{\circ} \mathrm{N}$ off the Portuguese continental slope: (a) R.R.S. Discovery (Jan. 1977); (b) Archival data (1914-1982). 
the MW outflow. The maximal salinity is as large as 36.4 psu. One can see a "tail" of less saline water spreading east of the lens. Fig. 7 shows a lens found in data gathered by R/V Ernst Krenkel (USSR) in August, 1983. The lens is positioned at the same latitude but 5 degrees west and is a part of the same branch of MW. In the case of more distant lens the salty "tail" is ultimately divorced from the lens body.

The distribution of individual lenses shown in Fig. 5 allows to trace the main cores and branches of MW in the Iberian Basin. The discrete analysis confirms the main paths of MW spreading found above using statistical methods. Particularly the presence of the southward branch of the southwest core is supported by occurrence of at least $10 \mathrm{MW}$ lenses. Additionally there are some indications that some lenses are transported across the Iberian abyssal plane far to the north (at $\left.42^{\circ}-44^{\circ} \mathrm{N}\right)$ by the weak northwest branch of the main MW outflow.

\section{Conclusions}

The joint analysis of the distribution of continuous and discrete component allows us to reveal the qualitative pattern of the MW outflow in the Iberian Basin (Fig. 8). The most important result of our study is that in the coastal zone of the Iberian Basin the climatic pattern of the MW spreading constructed here using historical data coincides basically with the synoptic pattern suggested by Zenk and Armi (1990) on the basis of CTD-measurements in January 1989 (F/S Meteor) and June 1989 (F/S Poseidon). This coincidence is not a chance. It is supported by the analysis of salinity distributions at $41^{\circ} \mathrm{N}$ (Fig. 9) and $42^{\circ} \mathrm{N}$ (Fig. 10) off the Portuguese continental slope. The synoptic sections conducted by R/V Elisha Kent Kane in November 1972 (Fig. 9a) and R.R.S. Discovery in January 1977 (Fig. 10a) are practically similar to the corresponding climatic sections (Figs. 9b, 10b) based on historical data collected in 1914-1982. It confirms our initial assumption that the Mediterranean Water distribution has a regular component in the eastern boundary layer of the Atlantic and the flow paths of MW are determined by local inhomogenities of the bottom topography.

\section{Acknowledgements}

Funds were provided by the Russian Fund of Fundamental Research grants 94-05-17302a and 94-05-17400a and by the Russian Ministry of Science and Technology and by ISF Grant JG 7100 .

\section{References}

Ambar, I. and Howe, M.R., 1979. Observations of Mediterranean outflow part 2 . The deep circulation in the vicinity of Gulf of Cadiz. Deep-Sea Res., 26: 555-568.

Armi, L. and Stommel, H., 1983. Four views of a portion of the North Atlantic Subtropical Gyre. J. Phys. Oceanogr., 13: 828-857.

Armi, L. and Zenk, W., 1984. Large lenses of highly saline Mediterranean Water. J. Phys. Oceanogr., 14: 1560-1576.

Armi, L., Hebert, D., Oakey, N., Price, J.F., Richardson, P.L. and Rossby, H.T., 1989. Two years in the life of Mediterranean salt lens. J. Phys. Oceanogr., 19: 354-370.

Belkin, I.M., Emelianov, M.V., Kostianoy, A.G. and Fedorov, K.N., 1986. Thermohaline structure of intermediate waters of the ocean and intrathermocline eddies. Intrathermocline eddies in the ocean. Inst. Oceanology, Moscow, pp. 8-34. (in Russian).

Belkin, I.M. and Kostianoy, A.G., 1988. Lenses of Mediterranean Water in the North Atlantic. Hydrophysical Investigations on the MEZOPOLYGON Program. Nauka, Moscow, pp. 110-123 (in Russian).

Berezutsky, A.V., Maximov, S.E., Rodionov, V.B. and Sklyarov, V.E., 1993. Peculiarities of intermediate Mediterranean Water's structure in the Canary Basin. Okeanologija, 33: 808-815 (in Russian).

Defant, A., 1955. Die Ausbreitung des Mittelmeerwassers im Nordatlantischen Ozean. Pap. Mar. Biolog. Oceanogr., 1: 465-470.

Dykhno, L.A., Morozov, E.G., Nikitin, S.V., Filyushkin, B.N. and Shilov, I.A., 1991. On Mediterranean Water lens destruction due to interactions with bottom topography. Okeanologija, 31: 55-61 (in Russian).

Elken, Yu., 1987. Lens-like eddies in the Baitic Sea and Atlantic Ocean. Proc. 3rd Meet. Sov. Oceanogr. Currents, synoptic and mesoscale eddies. Gidrometeoizdat, Leningrad, pp. 191-192 (in Russian).

Emelianov, M.V. and Fedorov, K.N., 1985. Structure and ransformation of intermediate waters of Mediterranean Sea and Atlantic Ocean. Okeanologija, 25: 206-214. (in Russian).

Filyushkin, B.N., 1989. Investigation of intrathermocline lenses 
of Mediterranean origin (16th cruise of R/V Vityaz, 03 June, 1988-16 Sept., 1988). Okeanologija, 29: 696-698. (in Russian).

Harvey, J., 1982. T-S relationship and water masses in the Eastern North Atlantic. Deep-Sea Res., 29: 1021-1033.

Hinrichsen, H.-H., Rhein, M., Käse, R.H. and Zenk, W., 1993. The Mediterranean water tongue and its chlorofluoromethanes signal in the Iberian Basin in early summer 1989. J. Geophys. Res., 98: 8405-8412.

Howe, M.R., Abdullah, M.I. and Deetae, S., 1974, An interpretation of the double $\mathrm{T}-\mathrm{S}$ maxima in the Mediterranean outflow using chemical tracers. J. Mar. Res., 32: 377-386.

Howe, M.R., 1982. The mediterranean water outflow in the Gulf of Cadiz. Oceanogr. Mar. Biol. Annu. Rev., 20: $37-64$.

Howe, M.R., 1984 Current and hydrographical measurements in the Mediterranean undercurrent near Cape St. Vincent. Oceanol. Acta, 7 : 163-168.

Katz, E.J., 1970. Diffusion of the core of Mediterranean water above the Mid-Atlantic Ridge crest. Deep-Sea Res., 17: 611-625.

Käse, R.H., Price, J.F., Richardson, P.L. and Zenk, W., 1986. A quasi-synoptic survey of the thermocline circulation and water mass distribution within the Canary Basin. J. Geophys. Res., 91: 9739-9748.

Käse, R.H. and Zenk, W., 1987. Reconstructed Mediterranean salt lens trajectories. J. Phys. Oceanogr., 17: 158163.

Käse, R.H., Beckmann, A. and Hinrichsen, H.-H., 1989. Observational evidence of salt lens formation in the Iberian Basin. J. Geophys. Res., 94: 4905-4912.

Kenelly, M.A., Prater, M.D. and Sanford, T.B., 1989. XBT and XSV data from the Gulf of Cadiz Expedition: R/V Oceanus Cruise 202. Tech. Rep. 8920, Appl. Phys. Lab., Univ. Washington, Seattle, $30 \mathrm{pp}$.

Koshliakov, M.N. and Panteleev, G.G., 1988. Thermohaline characteristics of a Meddy in tropical zone of North Atlantic. Hydrophysical investigations on the MEZOPOLYGON program. Nauka, Moscow, pp. 46-56. (in Russian).

Kostianoy, A.G. and Shapiro, G.I., 1989. On the forecast of lens-like eddy trajectories in the ocean. Dokl. Akad. Sci. USSR, 309: 1219-1222 (in Russian).

Krauss, W. and Käse, R.H., 1984. Mean circulation and eddy kinetic energy in the eastern North Atlantic. J. Geophys. Res., 89: 3407-3415.

Laanemets, J.J. and Lips, U.K., 1991. On the finestructure in Mediterranean salt lenses. Okeanologija, 31: 190-195 (in Russian).

Maltsev, N.E., Sabinin, K.D. and Furduev, A.V., 1990 An acoustic-oceanologic experiment at the lens of Mediterranean waters in the Atlantic Ocean. Akust. Zh., 36: 86-93 (in Russian).

Martins, C.G., 1992. Mediterranean water outflow revealed from historical data. Proc. Int. Workshop Outflows and Overflows in the Atlantic and their Role in the Eastern Boundary Current System. Univ. Lisbon, pp. 11-12.

Piip, A.T., 1969. Large cells of Mediterranean Water in the
Madeira-Canaries region. EOS (Trans. Am. Geophys. Union), 50: 193.

Pingree, R.D. and Le Cann, B., 1993. A shallow Meddy (a smeddy) from the secondary Mediterranean salinity maximum. J. Geophys. Res., 98: 20,169-20,185.

Plakhin, E.A. and Smirnov, V.G., 1982. Mediterranean Water in the central Atlantic. Okeanologija, 22: 569-573 (in Russian).

Prater, M. and Sanford, T.B., 1992. Dynamical aspects of the Cadiz Meddy and hypotheses of generation. Proc. Int. Workshop Outflows and Overflows in the Atlantic and their Role in the Eastern Boundary Current System. Univ. Lisbon, pp. 30-35.

Reid, J.L., 1978. On the middepth circulation and salinity field in the North Atlantic Ocean. J. Geophys. Res., 83: 5063-5067.

Richardson, P.L., Walsh, D., Armi, L., Schröder, M. and Price, J.F., 1989. Tracking three Meddies with SOFAR floats. J. Phys. Oceanogr., 19: 371-383.

Richardson, P.L., McCartney, M.S. and Maillard, C., 1991. A search for meddies in historical data. Dyn. Atmos. Oceans, 15: $241-265$.

Roemmich, D. and Wunsch, C., 1985. Two transatlantic sections: meridional circulation and heat flux in the subtropical North Atlantic Ocean. Deep-Sea Res., 32: 619-664.

Shapiro, G.I., Meschanov, S.L. and Emelianov, M.V., 1992. Mediterranean lens after collision with seamounts. Okeanologija, 32: 420-427 (in Russian).

Shultz Tokos, K.L., 1992. Preliminary RAFOS float observations from Meddy ASKA. Proc. Int. Workshop Outflows and Overflows in the Atlantic and their Role in the Eastern Boundary Current System. Univ. Lisbon, pp. 3640.

Stammer, D., Hinrichsen, H.-H. and Käse, R.H., 1991. Can Meddies be detected by satellite altimetry? J. Geophys. Res., 96: 7005-7014.

Swallow, J.C., 1969. A deep eddy off Cape St. Vincent. Deep-Sea Res. Suppl., V. 16: 285-296.

Thorpe, S.A., 1976. Variability of the Mediterranean undercurrent in the Gulf of Cadiz. Deep-Sea Res., 23: 711-727.

Vorobyov, V.N., Klyuikov, E.Yu., Ovchinnikov, E.M. and Provotorov, P.P., 1988. The thermohaline structure of a Mediterranean Water lens in the Canary Basin. Okeanologija, 28: 925-932 (in Russian).

Williams, R.T., Sarmiento, J.L., Rhines, P., Smethie, W.M. Jr., Rooth, C.G.H., Key, R.M., Takahashi, T. and Collins, C., 1986. Transient tracers in the ocean. Tropical Atlantic study, 1 December 1982-18 February 1983. Shipboard physical and chemical data report. Scripps Inst. Oceanogr. Tech. Rep. Ref. No. 86-16, 310 pp.

Zenk, W., Müller, T.J. and Wefer, G., 1989. BARLAVENTO-Expedition, Reise Nr. 9, 29.Dez.198817.Marz.1989. METEOR-Berichte, Univ. Hamburg, N 892, $238 \mathrm{pp}$.

Zenk, W. and Armi, L., 1990. The complex spreading pattern of Mediterranean Water off the Portuguese continental slope. Deep-Sea Res., 37: 1805-1823. 
Zenk, W., Klein, B. and Schröder, M., 1991. Cape Verde Frontal Zone. Deep-Sea Res., Suppl. V.38: 505-530.

Zenk, W., Shultz Tokos, K.L. and Boebel, O., 1992. New observations of Meddy movement south of the Tejo Plateau. Geophys. Res. Lett., 12: 2389-2392.

Zhurbas, V.M. and Lozovatsky, I.D., 1991. Lenses of Mediterranean origin near a chain of seamounts in the northeast
Atlantic. Lens-like eddies and fronts in the North-East Atlantic. Inst. Oceanology, Moscow, pp. 62-72 (in Russian).

Zubin, A.B. and Ozmidov, R.V., 1987. A lens of Mediterranean Water in the vicinity of the Ampere and Josephine seamounts. Dokl. Akad. Sci. USSR, 292: 716-719 (in Russian). 